Orthopäde 2013 · 42:449-452

DOI 10.1007/s00132-013-2110-4

Online publiziert: 19. Mai 2013

(c) Springer-Verlag Berlin Heidelberg 2013

T. Koller ${ }^{1} \cdot$ H. Luomajoki ${ }^{2}$

${ }^{1}$ Orthopädische und Handchirurgische Rehabilitation, Rehabilitationsklinik Bellikon

${ }^{2}$ Zürcher Fachhochschule für angewandte Wissenschaften, Zürich

\title{
Zweipunktediskrimination bei Phantomschmerzen
}

\author{
Effekt einer 4-wöchigen Therapie \\ bei einem oberarmamputierten \\ Patienten mit Phantomschmerzen
}

die kortikale Repräsentation normalisieren kann. Moseley et al. [7] sowie Moseley u. Wiech [8] belegten in Studien mit Patienten, die unter einem komplexen regionalen Schmerzsyndrom (CRPS) litten, dass taktile Stimulation alleine keinen Effekt hatte, mittels Diskriminationstraining hingegen eine deutliche Schmerzlinderung und Verbesserung der ZPD erreicht werden konnte. Die Patienten in der Gruppe mit taktiler Stimulation erhielten durch zweierlei Tastsonden taktile Impulse. Die Patienten in der Gruppe mit Diskrimination mussten anhand eines Fotos ihrer betroffenen Hand aufzeigen, wo und womit sie stimuliert wurden.

Luomajoki u. Moseley [5] verglichen bei Patienten mit unspezifischen chronischen Rückenschmerzen und Gesunden die Tastschärfe und die lumbosakrale motorische Kontrolle. Es zeigte sich, dass bei den Patienten im Vergleich zu den Gesunden die ZPD vergrößert und die motorische Kontrolle vermindert war.

50-90\% der Patienten mit Amputationen leiden an Phantommodalitäten $[1,4]$. Dieser Begriff beinhaltet den Phantomschmerz, das -gefühl und die -sensation. Phantomschmerz wird als anhaltender Schmerz im amputierten Körperteil definiert. Eine Phantomsensation beschreibt eine einschießende, schmerzhafte Sensation, die nur wenige Sekunden anhält. In der englischsprachigen Literatur wird der
Begriff der Phantomsensation definiert als jede Sensation des amputierten Glieds außer Schmerz [10]. Nicht-schmerzhafte Wahrnehmungen wie Position oder Schwellung des amputierten Körperteils, die in der englischsprachigen Literatur als nicht schmerzhafte Sensationen bezeichnet werden, sind im deutschsprachigen Raum als Phantomgefühl definiert $[11,12]$.

Ungeklärt bleibt, ob Phantommodalitäten gleich oder ähnlich wie der CRPSSchmerz auf die ZPD als Therapieintervention bzgl. kortikaler Reorganisation reagieren.

\section{Fallbeschreibung/Methodik}

Ein 47-jähriger Patient litt nach einer traumatischen Oberarmamputation (Autounfall) links vor 10 Wochen unter allen 3 Phantommodalitäten. Die Intensität des Phantomschmerzes betrug bei Befunderhebung mit der visuellen Analogskala (VAS) 4,125/10 (Mittelwert von 4 Messungen innerhalb $24 \mathrm{~h}$ ). Die Intensität des Phantomgefühls betrug 4,5/10 und die der Phantomsensationen 3,75/10. Der Nachtschlaf war nicht gestört. Die durchschnittliche Intensität aller Phantommodalitäten betrug am Erhebungstag 4,13.

Die Medikation des Patienten bestand aus Dichloranilinophenyl (75 mg 1-0-10) und Pregabalin (150 mg 1-0-1-0). Die Stumpflänge betrug ca. $10 \mathrm{~cm}$. Die Nar- wiesen sie nach, dass dieses Programm die Phantomschmerzen reduzieren und 


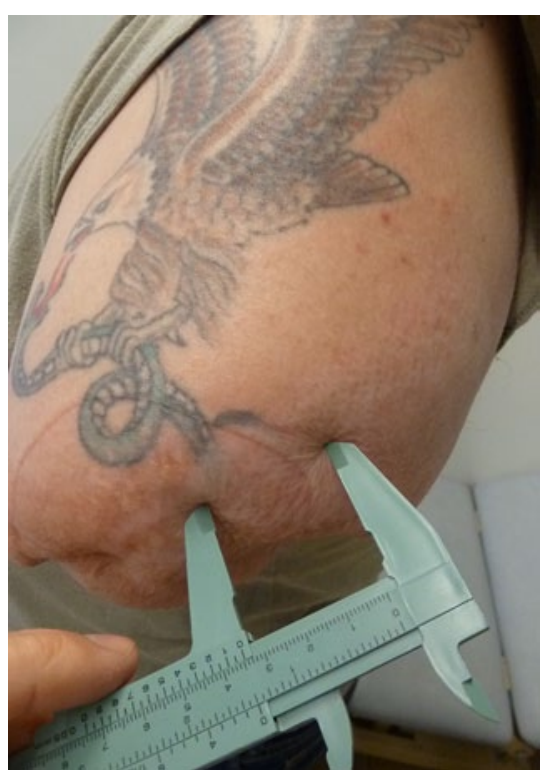

Abb. $1 \Delta$ Training der Diskriminationsfähigkeit

be war geschlossen, reizlos und gut verschieblich. Das Gewebe am Stumpfende war reizlos. Dorsal im Bereich der Spalthautdeckung waren noch etwas gerötete Stellen sichtbar. Im ventromedialen Bereich des Stumpfs war die Sensibilität leicht eingeschränkt (bei taktilem Stimulus ein leicht wattiges Gefühl), sonst überall intakt. Die Beweglichkeit des glenohumeralen Gelenks und der skapulothorakale Rhythmus waren unauffällig.

Zur Erfassung der 3 verschiedenen Phantommodalitäten wurde ein Schmerztagebuch mit einer VAS verwendet. Der Patient wurde aufgefordert, 4-mal täglich zu festgelegten Tageszeiten während der ganzen Testphase seine momentanen Phantommodalitäten zu dokumentieren. Die numerische Auswertung der Daten erfolgte mit einem transparenten Deckblatt, das mit einer Skala von 0 bis 10 versehen war. Der Diskriminationsabstand wurde mittels einer Schieblehre in Anlehnung an das Messprotokoll von Moberg [6] und Moseley et al. [7] gemessen.

Auf einem Foto des eigenen Oberarmstumpfs wurden die 4 Messstellen genau gekennzeichnet. Gemessen wurde am Stumpfende, im Schaftbereich lateral, außerhalb des Schaftbereichs lateral und am erhaltenen Arm auch außerhalb des Schaftbereichs lateral.

Für die Erfassung der funktionellen Einschränkungen wurde der Task-specific-scale-Fragebogen in Form einer nu-

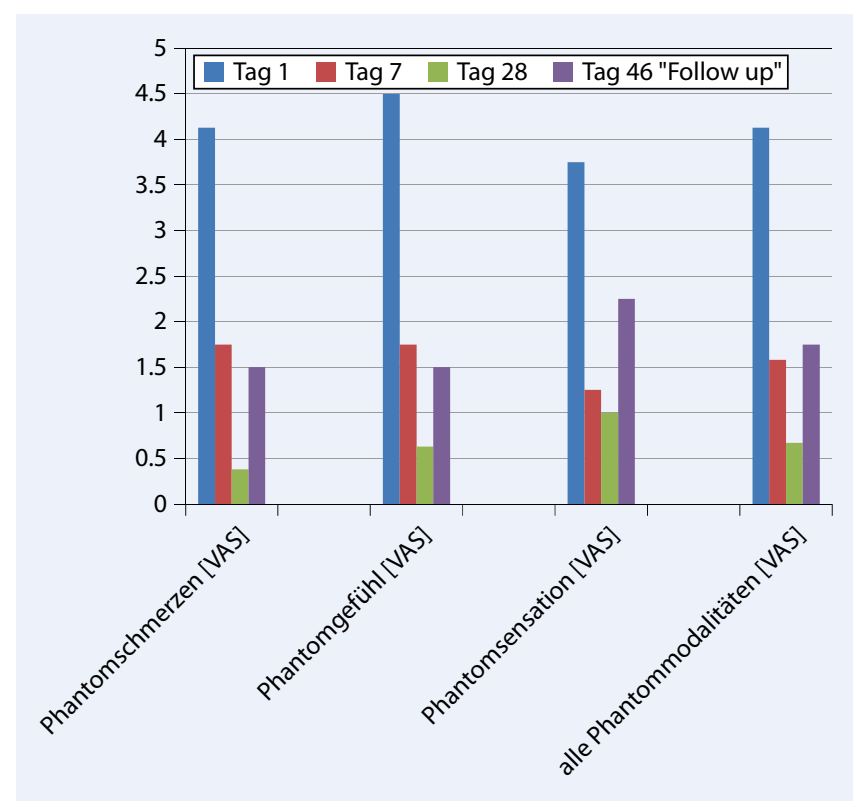

Abb. $2<$ Mittelwerte von Phantomschmerzen, -gefühl, sensationen und aller Phantommodalitäten zusammen. Vertikale Skalierung auf einer visuellen Analogskala (VAS) merischen Ratingskala (NRS) verwendet $[7,9]$. Dieser Fragebogen erfasst 5 selbstbestimmte Tätigkeiten des täglichen Lebens, Aktivitäten oder Funktionen, bei denen sich der Patient zurzeit eingeschränkt fühlt.

Bei der Befunderhebung betrug der Abstand der ZPD am Stumpfende 3,8 cm. Im Task-specific scale-Fragebogen erreichte der Patient einen Wert von 44 (50 würde bedeuten, dass der Patient keine der 5 Aktivitäten ausführen kann).

\section{Vorgehen/Datenerfassung}

Die Interventionseinheit bestand aus taktilen Stimuli mittels der beiden Spitzen einer Schieblehre, die abwechselnd mit einer oder beiden Spitzen gleichzeitig in verschiedenen Abständen im Bereich des Stumpfendes appliziert wurde (• Abb. 1; $[3,6])$. Der Patient wurde aufgefordert, jeweils über die Anzahl der wahrgenommen Spitzen und über den Ort des jeweiligen taktilen Stimulus Auskunft zu geben. Auf einem Foto vom eigenen Oberarmstumpf zeigte er die von ihm wahrgenommenen Stellen an. Der Patient konnte während den Interventionen seinen Stumpf nicht sehen [7]. Äußerst wichtig war bei der Intervention, dass der taktile Stimulus nicht als nozizeptiver Stimulus wahrgenommen wurde [5]. Vom Tag 2 bis einschließlich Tag 6 erhielt der Patient täglich 3 Interventionseinheiten von je- weils $10 \mathrm{~min}$. Die Einheiten waren möglichst gut über den Tag verteilt.

Am Tag 7 erfolgten die zweite Befunderhebung des Diskriminierungsabstands, eine neue Beurteilung mittels Task-specific-scale-Fragebogen und die Auswertung des Schmerztagebuchs der ersten Woche. Der Patient nahm in dieser Zeit (und auch schon 2 Wochen zuvor) am stationären Rehabilitationsprogramm teil; dieses bestand aus Einzelphysiotherapie (Erhalt der Beweglichkeit und Kräftigung des Stumpfs), Einzelergotherapie (Rekrutierung neuer motorischer Muster als Vorbereitung zur Steuerung der Armprothese), individueller Trainingstherapie zur Verbesserung von Propriozeption und Geschicklichkeit der erhaltenen oberen Extremität sowie allgemeinem Kraftund Ausdauertraining.

Während weiterer 3 Wochen fand nur noch einmal täglich eine 10-minütige Intervention der ZPD statt. Das beschriebene stationäre Rehabilitationsprogramm blieb bestehen. Um einen etwaigen Effekt der Intervention zu erkennen, wurde während und vor der ganzen Testphase bewusst auf die Spiegeltherapie und das Recognizeprogramm verzichtet $[1,2]$.

\section{Resultate}

Der Mittelwert aller Phantommodalitäten wurde am Tag 1 mit 4,13/10 erhoben. Am Tag 28 betrug dieser Wert noch 0,67/10. 
Der Mittelwert aller Phantommodalitäten nahm kontinuierlich ab (• Abb. 2). Auffallend war, dass sich der Phantomschmerz während der Testphase von den 3 erhobenen Phantommodalitäten am meisten reduzierte. Die Phantomsensationen veränderten sich am wenigsten; der Patient reagierte am längsten mit Phantomsensationen sensitiv auf das Autofahren.

Das Absetzen aller Medikamente am Tag 11 zeigte seine Wirkung am Tag 12 durch einen klar erhöhten Mittelwert (3,21/10) aller Phantommodalitäten. Dieser erhöhte Mittelwert normalisierte sich aber nach 3 Tagen.

Bei der Follow-up-Messung am Tag 46 war der Mittelwert aller Phantommodalitäten wieder auf 1,75/10 angestiegen. Der Abstand der ZPD veränderte sich nur am Stumpfende und im Schaftbereich lateral (• Tab. 1). Diese 2 Stellen wurden auch während der Testphase mit ZPD stimuliert. Am Stumpfende veränderte sich der ZPD-Abstand von 3,8 cm (Tag 1) auf 2,7 cm (Tag 28; - Tab. 1).

\section{Diskussion}

Diese Kasuistik gibt Hinweise darauf, dass die taktile Stimulation (im Sinne der ZPD) mit gleichzeitiger topographischer Zuordnung auf einem Stumpffoto den Phantomschmerz bzw. die 3 Phantommodalitäten positiv beeinflussen kann [7]. Die erhobenen Resultate könnten Hinweise auf eine Normalisierung der kortikalen Repräsentation geben. Moseley et al. [7] sowie Moseley u. Wiech [8] belegten in Studien mit CRPS-Patienten, dass taktile Stimulation alleine keinen Effekt hatte, mit einem Diskriminationstraining aber eine deutliche Schmerzlinderung und Verbesserung der ZPD erreicht werden konnte.

Offen bleibt, ob der Abstand der ZPD wirklich mit dem Rückgang aller Phantommodalitäten korreliert oder dies $\mathrm{Zu}$ fall ist. In unserer Rehabilitation war ein Prothesentraining leider nur bedingt durchführbar, weil es beim Anpassen der Prothese Schwierigkeiten gab (empfindliche, schmerzhafte Stellen). Am Ende der stationären Rehabilitation betrug die Tragezeit dieser Prothese ca. 4-6 h täglich. Inwieweit das Tragen der Prothe-

Orthopäde 2013 · 42:449-452 DOI 10.1007/s00132-013-2110-4

(c) Springer-Verlag Berlin Heidelberg 2013

\section{T. Koller $\cdot$ H. Luomajoki}

Zweipunktediskrimination bei Phantomschmerzen. Effekt einer 4-wöchigen Therapie bei einem oberarmamputierten Patienten mit Phantomschmerzen

\section{Zusammenfassung}

Es gibt erste Evidenzen dafür, dass Phantomschmerzen mit einer gestörten Organisation des sensorischen Kortex einhergehen und diese Organisation mit einem Training der Zweipunktediskrimination (ZPD) normalisiert werden könnte. In dieser Kasuistik konnte bei einem oberarmamputierten Patienten während einer 28-tägigen Testphase mittels ZPD eine Reduktion aller 3 Phantommodalitäten (Phantomschmerz, -gefühl und -sensation) von $\mathrm{m}=4,13 / 10$ (visuelle Analogskala, VAS) auf 0,67/10 beobachtet werden. Die Lebensqualität und die Leistungsfähigkeit konnten deutlich gesteigert werden. Dies kann ein vielversprechender Hinweis auf eine bessere gesellschaftliche und arbeitsbezogene Reintegration sein.

\section{Schlüsselwörter}

Amputation · Kortikale Repräsentation . Intervention · Lebensqualität .

Leistungsfähigkeit

\section{Two-point discrimination for phantom pain. Effect of a 4-week therapy in an upper arm amputee with phantom pain}

\begin{abstract}
There is evidence that phantom pain is associated with a disrupted organization of the sensory cortex and that this organization can be normalized by training with two-point discrimination (TPD). In this case study a reduction in all three phantom modalities (i.e. phantom pain, phantom feeling and painful phantom sensation) and a reduction in pain level from $m=4.13 / 10$ visual analogue scale (VAS) to $m=0.67 / 10$ (VAS) could be observed
\end{abstract}

in a patient with an upper limb amputation during a test period of 28 days with TPD. The quality of life and performance increased significantly. This can be a promising indication for a better social and work reintegration.

\section{Keywords}

Amputation - Cortical representation . Intervention · Quality of life · Perfomance se im Alltag die Schmerzsituation positiv beeinflusste, bleibt in dieser Kasuistik offen.

Überraschend war, dass sich die Phantommodalitäten nach einer Phase mit konstanten Schmerzen von ca. 2 Monaten Dauer reduzierten [2, 4]. Für ein überwiegend chronifizierendes Geschehen sprachen in diesem Fall der geringe Effekt der Schmerzmedikamente Dichloranilinophenyl und Pregabalin und die konstante Phantomschmerzproblematik seit der Amputation. Klar gegen ein chronifizierendes Geschehen sprachen die differenzierten Phantomangaben des Patienten sowie das belastungsund aktivitätsabhängige Auftreten und der schnelle Rückgang der Phantommodalitäten.

Wenn die ZPD als Therapieintervention bei der kortikalen Repräsentation den gleichen Effekt hat wie die transku- tane Nervenstimulation in der Studie von Flor et al. [3], könnte es auch sein, dass die ZPD auch bei anderen armamputierten Patienten eine Reduktion der Phantommodalitäten zur Folge hat.

Der in dieser Kasuistik beschriebene Effekt ist sehr erfreulich, da es im Einzelfall gelang, innerhalb kurzer Zeit die Phantommodalitäten eines $\mathrm{Pa}$ tienten deutlich und relevant $\mathrm{zu}$ mindern. Die Lebensqualität und die Leistungsfähigkeit konnten beim Patienten dadurch deutlich gesteigert werden (- Tab. 2). Dies kann eine vielversprechende Grundlage für eine leichtere gesellschaftliche und arbeitsbezogene $\mathrm{Re}$ integration sein. 
Tab. 1 Veränderung des Abstands der Zweipunktediskrimination (ZPD) während der Testphase und beim Follow-up am Tag 46

\begin{tabular}{lllll}
\hline Messprotokoll & 1. Messung & 2. Messung & 3. Messung & 4. Messung \\
\hline ZPD $(\mathbf{c m})$ & Tag $\mathbf{1}$ & Tag 7 & Tag 28 & Tag 46 \\
\hline Stumpfende & 3,8 & 3,1 & 2,7 & 2,8 \\
\hline Schaftbereich lateral & 4,8 & 4,3 & 3,7 & 4 \\
\hline Außerhalb Schaftbereich & 4,7 & 4,8 & 5 & 4,4 \\
\hline Erhaltener Arm proximal & 4,8 & 4,8 & 5,5 & 4,6 \\
\hline
\end{tabular}

Tab. 2 Resultate aus dem Task-specific scale-Fragebogen

\begin{tabular}{lllll}
\hline Aktivität (NRS 0-10) & Tag 1 & Tag 7 & Tag 28 & Tag 46 \\
\hline Seitenlage links & 10 & 10 & 10 & 3 \\
\hline Radfahren & 6 & 6 & 0 & 0 \\
\hline Frühstücksbrot zubereiten & 10 & 10 & 0 & 0 \\
\hline Fleisch schneiden & 10 & 10 & 0 & 0 \\
\hline Duschen & 8 & 8 & 0 & 0 \\
\hline NRS numerische Ratingskala. & & & &
\end{tabular}

\section{Fazit}

Die Verbesserung der Körperwahrnehmung mittels ZPD-Training könnte eine gute Möglichkeit sein, Patienten mit Phantomschmerzen nach einer Amputation zu helfen.

\section{Korrespondenzadresse}

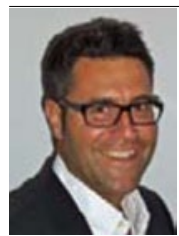

\section{T. Koller}

Orthopädische und

Handchirurgische

Rehabilitation,

Rehabilitationsklinik Bellikon

CH-5454 Bellikon

Schweiz

thomas.koller@rehabellikon.ch

Interessenkonflikt. Der Autor gibt für sich und seinen Koautor an, dass kein Interessenkonflikt besteht.

\section{Literatur}

1. Diers M, Christmann C, Koeppe C et al (2010) Mirrored, imagined and executed movements differentially activate sensorimotor cortex in amputees with and without phantom limb pain. Pain 149(2):296-304. doi:10.1016/j.pain.2010.02.020

2. Flor $\mathrm{H}(2000)$ The functional organization of the brain in chronic pain. Prog Brain Res 129:313-322. doi:S0079-6123(00)29023-7

3. Flor H, Denke C, Schaefer M, Grüsser S (2001) Effect of sensory discrimination training on cortical reorganisation and phantom limb pain. Lancet 357(9270):1763-1764. doi:10.1016/s01406736(00)04890-x

4. Kern U, Busch V, Rockland M et al (2009) Prävalenz und Risikofaktoren von Phantomschmerzen und Phantomwahrnehmung in Deutschland. Schmerz 23:479-488. doi:10.1007/s00482-009-0786-5
5. Luomajoki H, Moseley G (2010) Tactile acuity and lumbopelvic motor control in patients with back pain and healthy controls. $\mathrm{Br} \mathrm{J}$

6. Moberg E (1990) Two-point discrimination test. A valuable part of hand surgical rehabilitation, e. $\mathrm{g}$. in tetraplegia. Scand J Rehabil Med 22(3):127-134

7. Moseley GL, Zalucki NM, Wiech K (2008) Tactile discrimination, but not tactile stimulation alone, reduces chronic limb pain. Pain 137(3):600-608 doi:10.1016/j.pain.2007.10.021

8. Moseley GL, Wiech K (2009) The effect of tactile discrimination training is enhanced when patients watch the reflected image of their unaffected limb during training. Pain 144(3):314-319. doi:10.1016/j.pain.2009.04.030

9. Westaway MD, Stratford PW, Binkley JM (1998) The patient-specific functional scale: validation of Sports Phys Ther 27:331-338

10. Hill A (1999) Phantom limb pain: a review of the literature on attributes and potential mechanisms. J Pain Symptom Manage 17(2):125-142. doi:S0885392498001365 [pii]

11. Kern U, Busch V, Rockland M et al (2009) Prävalenz und Risikofaktoren von Phantomschmerzen und Phantomwahrnehmung in Deutschland. Schmerz 23:479-488. doi:10.1007/s00482-009-0786-5

12. Steffen $P$ (2006) Phantom limb pain. Anasthesiol Intensivmed Notfallmed Schmerzther 41(6):378386. doi:10.1055/s-2006-947304 (quiz 387-378)

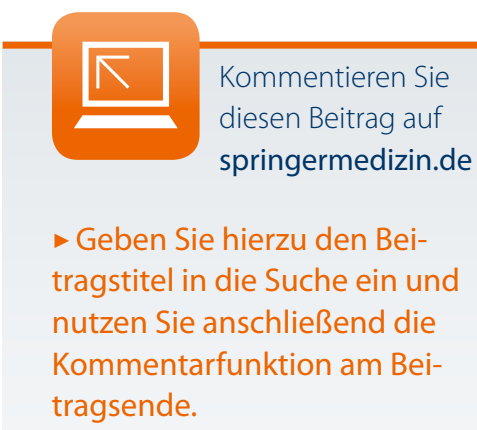
Sports Med. doi:bjsm.2009.060731 [pii]10.1136/ bjsm.2009.060731 its use in persons with neck dysfunction. J Orthop

\section{AE-Preis 2013}

Die Arbeitsgemeinschaft Endoprothetik schreibt den AE-Preis 2013 als Auszeichnung für eine innovative wissenschaftliche Arbeit auf dem Gebiet der Endoprothetik aus.

Teilnahmeberechtigt sind Orthopäden und Unfallchirurgen und alle Mitglieder der AE und $A E-C o m G e n$. Eingereicht werden können ausschließlich Arbeiten, die in einem peer reviewed Journal publiziert oder zur Publikation angenommen worden sind. Die Publikation darf nicht älter als ein Jahr sein. Es werden nur Arbeiten berücksichtigt, die nicht gleichzeitig für einen anderen Preis eingereicht oder bereits anderweitig ausgezeichnet worden sind.

Die Dotierung beträgt EUR 10.000,--.

Die Arbeiten müssen in deutscher oder englischer Sprache abgefasst sein und in 6-facher Ausfertigung bis zum

\subsubsection{3}

bei der Geschäftsstelle der Arbeitsgemeinschaft Endoprothetik e.V., Oltmannsstraße 5, 79100 Freiburg, eingegangen sein. Die eingereichten Arbeiten werden von einer unabhängigen Jury bewertet.

Beim 15. AE-Kongress am 06./07. Dezember 2013 in München sollte die/der Preisträger/in in einem Referat einen Überblick über die Arbeit geben.

Der Rechtsweg ist ausgeschlossen.

Weitere Informationen unter

Tel. 0761 / 4564 - 7666,

www.ae-germany.com

Quelle:

Arbeitsgemeinschaft Endoprothetik e.V. 